\section{ADDISON'S DISEASE, A SPECTRUM OF PRESENTATION}

\author{
T. Bourke, D. Carson \\ Royal Belfast Hospital for Sick Children, \\ Belfast, UK
}

Background: We reviewed the spectrum of clinical presentation of Addison's disease in order to raise awareness of atypical presentations and chronic features.

Methods: All patients with a diagnosis of Addison's disease attending our hospital over a fifteen-year period were identified. A retrospective chart review was carried out. Details of acute and chronic presenting features and initial investigations were recorded.

Results: 12 patients were identified and 11 charts located. There were 7 males and 4 females with a median age at diagnosis of 8.3 years. 7 patients presented with an adrenal crisis following an acute illness. These ranged in severity from a short episode of vomiting to a VF arrest. All of these patients had hyponatraemia (106 to $127 \mathrm{mmol} / \mathrm{l}$ ); however only 5 had a hyperkalaemia (5.6 to $7.6 \mathrm{mmol} / \mathrm{l})$ and only 1 had documented hypoglycaemia. 5 had identifiable chronic features including weight loss, lethargy, postural hypotension, poor school performance and hyperpigmentation. 2 had previously been reviewed at an out-patient department with regard to these problems. 4 of the 11 patients received a diagnosis of Addison's disease without suffering an adrenal crisis. Their presenting features included pigmentation of a scar, unexplained chronic hyponatraemia, family history and associated endocrinopathies. 2 of the male patients had elevated VLCFAs leading to a subsequent diagnosis of adrenoleucodystrophy.

Discussion: Most acute clinicians are aware of the classical presentation of an adrenal crisis. Knowledge of atypical presentations and the chronic features of adrenal insufficiency could facilitate earlier diagnosis and potentially avoid life threatening adrenal crises.

\section{8}

\section{THE ENDOCRINE DISTURBANCES IN OBESES CHILDREN OF SOUTH WEST OF ROMANIA}

O.D. Marginean ${ }^{1}$, I.D. Simedrea ${ }^{2}$, M.A. Lesovici ${ }^{3}$, D.M. Bucuras ${ }^{4}$, T.I. Marcovici ${ }^{5}$, C.M. Gug ${ }^{6}$

${ }^{1}$ Endocrinology, ${ }^{2}$ Gastroenterology, University of Medecine and Pharmacy 'Victor Babes ', ${ }^{3}$ Laboratory, Clinical Children Hospital 'Louis Turcanu', "Endocrinology, ${ }^{5}$ Pediatrics, ${ }^{6}$ Genetics, University of Medicine and Pharmacy 'Victor Babes', Timisoara, Romania

Bacground :The adult obesity is associated with endocrine, metabolic and mecanical complications but in children obesity the endocrine disturbances are not well know.

Matherial and method: Between 2004-2009 we study the child atmetted in the Ist Padiatric Clinical $^{\text {st }}$ Children Hospital "Louis Turcanu” Timisoara from the south west of the country, by anamnesis, clinical examinations and antropomeric parameters (body weith, heigth, abdominal folder), BMI. The pubery stages were registered according to Tanner criterias. We follow-up of the, metabolic and endocrine complicatios (hyperinsuinemia, dislipidemia, hypercolesterolemia, HOMA index- insulinoresistance, hypercotisolism, early adrenarche, hyperprolactinemia, Sex hormon binding protein -SHBG level).

Results: During the study, we found 7135 obeses child $(56,69 \%)$, mostly boys 3722 . A 422 of them were infants. The majority of child got obesity by high calories intake only 16 had genetic obesity (Prader Willy, Laurence- Bardet- Bield ). We found insulinoresistence in $46 \%$ of the teenagre obeses and $32 \%$ of the children. We found a good corelations between the insulinoresitence and low level of SHBG. In 27 girls we found early adrenarche; 15 PCOS, in 4 cases diabetis mellitus type 2; the mecanical complications were present in all children. All tenaager patients have depression, isolations and low performance in school. We found a hight percentage of uniparental family.

\section{Conclusions:}

1. In the last yares the incidence of child obesity rise darmaticaly in our region

2. The incidence of endocrine disturbances in obeses children in relative height. 
3. The magnitude of this are in direct relationship with the degree of obesity and age.

\section{9}

\section{OBESITY-THERAPY AND SELF-REGULATION IN CHILDREN FROM AGE 8 TO 17 YEARS OVER 10 YEARS}

\author{
A. Chen-Stute ${ }^{1}$, M. Pinnow ${ }^{2}$, S. Kopczynski², \\ U. Faupel ${ }^{3}$
}

${ }^{1}$ Ev. Bethesda-Johanniter-Klinikum, Adipositas-

Zentrum, Duisburg, ${ }^{2}$ Ruhr-Universität-Bochum, Bochum, ${ }^{3}$ Adipositas-Zentrum, Duisburg, Germany

Background and aims: Since 1997 we carry out the treatment program for children from age 8-12 years and adolescents from age 13-17 years with their parents. The goal is to learn self-control and self-regulation of eating behaviour, to obtain better physical fitness and slithly bodyweight reduction.

Methods: Because the psycho-social problems are often overlooked and focusing on the longterm physical health, there is an emerging evidence to show the association between the psychological problems and obesity of the children and adolescents.Our obesity-therapy (>P.97) is concipatied over 3 years for children and parents. The program includes in the first year 1 times / week education in behaviour modification and nutrition by a psychologist and nutrition by a psychologist and nutritionist, the same happens with the parents in a way of family-therapy. The children participate in 3-times a week of different physical fitness in the first year.

Results: In an on-going-study we can report over 400 children of our therapy-Program that based on the 7-Phase-Mode of Kanfer. After 3 years there is an reduction of BMI-SDS $2,7 \pm 0,5 \mathrm{~kg} / \mathrm{m}^{2}$ to $2,1 \pm 0,8$ $\mathrm{kg} / \mathrm{m}^{2} .43 \%$ have a reduction of 0,2 and $29 \%$ of BMI-SDS $<0,5.72 \%$ of all children and adults have positive results.

Conclusion: The results of the T.O.M.-program indicate that long term weight-control is feasible in children and adolescents and is worthwhile as a wide range of improvements in risk faktors and selfconfidence can be demonstrated.
1110

\section{ECTOPIC NEUROHYPOPHYSIS - CASE REPORT}

\author{
N. Ferraria ${ }^{1}$, P. Afonso ${ }^{2}$, S. Castro ${ }^{2}$, D. Amaral ${ }^{3}$, \\ L. Lopes ${ }^{4}$ \\ ${ }^{1}$ Pediatric Department, ${ }^{2}$ Hospital Nossa Senhora \\ do Rosário, Centro Hopitalar Barreiro- Montijo, \\ Barreiro, ${ }^{3}$ Pediatric Department, ${ }^{4}$ Hospital Dona \\ Estefânia, Centro Hospitalar Lisboa Central, \\ Lisboa, Portugal
}

Background: Ectopic Neurohypophysis is a rare congenital anomaly of the pituitary gland associated with growth hormone and other anterior pituitary hormone deficiencies.

Aims: To report a case of hypopituitarism due to ectopic neurohypophysis presenting with hypoglycemic seizures.

Case report: A 7-year-old Romanian boy was admitted to our hospital with a generalized tonicclonic seizure associated with severe hypoglycemia $(25 \mathrm{mg} / \mathrm{dL})$. His past medical history was remarkable for a perinatal history of asphyxia; congenital left eye strabismus; and three previous episods of hypoglicemic seizures with inconclusive metabolic and neurologic investigations. He was under treatment with carbamazepine for 3 months.

According to his mother he had also language delay and growth retardation.

The physical examination revealed a boy with a hoarse tone voice, dry skin, brittle hair, cold extremities and left eye convergent strabismus. His height was in the $3^{\text {rd }}$ percentile (target height between $75^{\text {th }}-85^{\text {th }}$ percentile).

Laboratory studies showed hypercholesterolemia, normal values of ACTH, cortisol and TSH but abnormal low values of free T4 and IGF-1. Antithyroglobulin and antiperoxidase antibodies were negative.

Abdominal and thyroid ultrasounds were normal. Left wrist X-ray revealed a bone age of 4 years.

The brain MRI showed an ectopic neurohypophysis. The pituitary stalk was not seen.

The patient started thyroid hormone replacement therapy and has been proposed for recombinant human growth hormone. 